\title{
Upcoming Events in Pediatric Cardiology
}

C Springer Science+Business Media, LLC, part of Springer Nature 2022

Please note that due to Covid-19 pandemic, many meetings are being held virtually. Please check organization websites for details

February 16-20, 2022

Cardiology 2022: The New Normal-Transformation in Pediatric and Congenital Heart Care

Huntington Beach, CA

https://chop.cloud-cme.com/course/courseoverview?P= $5 \& \mathrm{EID}=2646$

February 17-19, 2022

CATCH 2022: Caring for Adults and Teens with Congenital Heart Disease

Four Seasons Resort Oáhu at Ko Olina, Hawaií

https://www.hawaiipacifichealth.org/CATCH

February 21-23, 2022

WASP (Write a Scientific Paper)

International, intense 3-day courses

18 WASP COURSES HELD SINCE 2010

Malta, \& online

https://www.ithams.com/wasp/

February 26-March 1, 2022

Cardiovascular Research Technologies CRT22

Washington, DC

https://crtmeeting.org/attend-crt/about-crt

June 23-25, 2022

\section{9th Annual ACHA Conference}

Bloomington, $\mathrm{MN}$

https://www.achaheart.org/get-involved/events/2022/9thnational-conference/

April 29-May 1, 2022

Heart Rhythm 2022

San Francisco, CA,

https://heartrhythm.com/
May 6, 2022

Controversies in Cardiac Arrhythmias CICA 2022

Washington DC

https://cica-ep.org/index.html

June 23-25, 2022

9th Annual ACHA Conference

Bloomington, $\mathrm{MN}$

https://www.achaheart.org/get-involved/events/2022/9thnational-conference/

June 22-25, 2022

CSI Frankfurt

Frankfurt, Germany

https://www.csi-congress.org/conferences-courses/confe rences/csi-frankfurt

July 12-16, 2022

11th Congress of the World Federation of Pediatric Intensive \& Critical Care Societies

Cape Town, South Africa I Online \& Onsite

https://wfpiccs.org/wfpiccs-2022/?mkt_tok=MzA1LVFVSy 01MTkAAAGAinMZ-HgtnjZgWDYQbucYhWQIaXpfqL wAGrjy7gkPdgFY04sg7HNLCGQ9qsQxgBYJbK4SI5RwzJM THLlaDwgDQIwlgWTNZ0AraIk5PPkW4SsU

September 7-10, 2022

Pediatric and Congenital Interventional Cardiology Symposium PICS

Chicago and online

https://www.picsymposium.com/

August 27-September 1, 2023

8th World Congress of Pediatric Cardiology and Cardiac Surgery Washington, DC

http://www.cvent.com/events/world-congress-of-pedia tric-cardiology-and-cardiac-surgery/event-summary-34277 6c3ba39428eb9986e6f72d69b65.aspx

Publisher's Note Springer Nature remains neutral with regard to jurisdictional claims in published maps and institutional affiliations. 